\section{Ocular disorders in children with Down syndrome}

\section{Siegfried M. Pueschel}

Child Develoment Center

Department of Pediatrics

\section{Stefan Gieswein}

Department of Ophthalmology

\author{
Rhode Island Hospital \\ Brown University School of Medicine \\ Providence, Rhode Island, U.S.A.
}

\begin{abstract}
Seventy-three patients with Down syndrome between the ages of 5 to 18 years were initially enrolled in this study and there were 68 patients in the final sample. Information was obtained from previous ophthalmologic examinations and parents completed a questionnaire pertaining to ocular disorders. Subsequently, the patients' visual acuity was assessed using Snellen or Kindergarten Test Charts for far vision testing and the Rosenbaum Pocket Vision Screener or the Child's Recognition and Near Point Test for near vision screening. A select group of children underwent a detailed ophthalmologic examination. The results of the parent questionnaire and data from the initial ophthalmologic screening are presented in Table 1 and 2, respectively. Results obtained from screening as well as from ophthalmologic evaluations indicate that 12 of 68 patients had bilateral poor vision $(20 / 50$ or below) and 15 patients were found to be amblyopic. Five of the 15 children with amblyopia had associated strabismus, another five had anisometropic amblyopia, two had both strabismus and anisometropia and three patients had no associate findings. This study suggests that children with Down syndrome may be at a greater risk for visual impairment than previously reported and that many of them may have amblyopia. Therefore, it is important that these children be examined ophthalmologically at regular intervals and treated appropriately if a visual disorder has been identified.
\end{abstract}

\section{Introduction}

(C) 1993,1999. The Down Syndrome Educational Trust Down Syndrome Research and Practice 1993, 1 (3) 129-132
The eyes, and features surrounding the eyes of the person with Down syndrome have attracted interest by many physicians since the mid 1800's (Down 1866; Sequin 1866; Tredgold 1908; Brushfield 1924; Lowe 1949). Down (1866) already had observed that "the eyes are obliquely placed, and the internal canthi are more than normal distance from one another," and "The palpebral fissure is very narrow." In addition to the external ocular features there are numerous other ocular manifestations in individuals with Down syndrome including strabismus, nystagmus, keratoconus, cataracts, hyperplasia of the iris, and refractive errors (Catalano, 1992). Many of the latter mentioned ocular concerns are occurring at a higher frequency in persons with Down syndrome when compared with those who do not have this chromosomal disorder (Pueschel, 1981).

In reviewing the multitude of reports on ophthalmologic disorders in persons with Down syndrome, it is surprising that the prevalence of amblyopia in persons with Down syndrome has never been investigated, since it is well known that there is an increased prevalence of structural abnormalities, strabismus, refractive errors, and other ophthalmologic disorders in individuals with Down syndrome which potentially could cause amblyopia. For example, Caputo, Wagner and Reynolds (1989) examined 187 patients with Down syndrome and found that $57 \%$ of the study individuals had strabismus. Hiles, Hoyme and McFarlane (1974) noted that $34 \%$ of 123 patients with Down syndrome had either esotropia or exotropia. Also, Gnad and Rett (1979) observed strabismus to occur at a higher frequency (31\%) in 420 patients with Down syndrome. Moreover, many reports in the literature indicate that persons with Down syndrome have refractive errors; approximately $50 \%$ are myopic and $15 \%$ to $20 \%$ are hyperopic (Pueschel, 1981).

In spite of the increased prevalence of various visual concerns which are often associated with amblyopia, this ocular disorder has not been studied in detail in the Down syndrome population. There are only two reports in the literature which make reference to amblyopia in persons with Down syndrome. Hiles, Hoyme and McFarlane (1974) noted that 11 out of 123 (8.5\%) patients with Down syndrome had amblyopia and Jaeger (1980) observed amblyopia in 12.5\% of patients with Down syndrome who had ocular misalignment.

\section{Patients and Methods}

Children enrolled in this study were recruited from the Down Syndrome Program of the Child Development Center at Rhode Island Hospital. Since the Child Development Center is the only facility providing comprehensive care for children with Down syndrome in Rhode Island and since the Child Development Center has a close relationship with the two cytogenetic laboratories in this state, almost complete ascertainment of persons with Down syndrome is obtained and there is no known selection bias.

Initially, we sent 106 letters to parents of children with Down syndrome between the ages of 5 and 18 briefly discussing the intent, nature, and importance of this study. Parents who agreed that their child participate in this investigation were invited to come to the Child Development Center where we explained in more detail the procedures to be carried out. When parents and child indicated that they would like to take part in this study, they were asked to sign an informed consent form. 
Of the original 106 families contacted, 73 were enrolled in the study. The remaining 33 families either had moved out of state, their address was unknown or incorrect, their children were severely mentally retarded and were unable to participate or the parents refused that their child be part of the study.

With the parents' permission information was obtained from previous ophthalmologic examinations. Parents were asked to complete a brief questionnaire pertaining to ocular disorders, previous ophthalmologic investigations, corrections of refractive errors, and other visual concerns of their children. During the subsequent screening, the children's eyes were examined and their visual acuity was assessed by the participating pediatrician who had special training in performing these procedures. The Snellen or Kindergarten Test Charts for far vision testing and the Rosenbaum Pocket Vision Screener or the Child's Recognition and Near Point Test were used following standard testing procedures. Although refractive errors can be identified without the active co-operation of the subject, for this study - in order to be consistent - we choose the above described method.

After the screening, children were reinvited for a more thorough ophthalmologic examination if the results of the screening differed markedly from that of previous ophthalmologic assessments, if there was bilateral poor vision, if a significant discrepancy between the right eye's visual acuity and the left eye's visual acuity was observed, if the result of the screening was uninterpretable or if any other significant ocular findings were noted. These follow up examinations were performed by well-trained ophthalmologists. The examination included evaluation of ocular motility, strabismus, globe, ocular adnexa, and the fundus. Snellen letters, numbers, or pictures charts were used to assess visual acuity. An examination of central, steady, maintained (CSM) visual behaviour was done in very young children and those with severe mental retardation.

For this study amblyopia was defined as having a significant visual loss not associated with fundoscopic abnormalities and not correctable with refraction and when a difference of two Snellen acuity lines between right and left eyes or if unilateral CSM vision and a clear preference for fixation in one eye was present.

\section{Results}

The evaluation of the parent questionnaires revealed that 58 of the 73 patients had one or more examinations done by their private ophthalmologist. Parents often did not know whether their child was myopic or hyperopic. Of the 73 youngsters with Down syndrome 26 were wearing glasses. Parents of 59 children had observed that their child was usually sitting very near to the television set while watching a program. Other findings as reported by the parents are listed in Table 1.

The results of the initial ophthalmologic screening are shown in Table 2. It is of note that a large number of children have strabismus: 27 children were found to have esotropia and 9 have exotropia.

During the initial visual acuity screening of the 73 individuals with Down syndrome, 12 did not co-operate well. These youngsters often were young (5 to 8 years), some were severely mentally retarded, and/or displayed hyperactive or

\begin{tabular}{|l|c|c|c|}
\hline \multicolumn{4}{|c|}{ Results of Parents' Questionnaire } \\
\hline & Yes & No & $\begin{array}{c}\text { Do Not } \\
\text { Know }\end{array}$ \\
\hline Blepharitis & 31 & 40 & 2 \\
\hline Nystagmus & 11 & 56 & 6 \\
\hline Strabismus & 33 & 40 & 0 \\
\hline Cataracts & 2 & 62 & 9 \\
\hline Myopia & 15 & 24 & 34 \\
\hline Hyperopia & 12 & 26 & 35 \\
\hline Glasses & 26 & 44 & 3 \\
\hline Astigmatism & 5 & 36 & 32 \\
\hline Corneal disorders & 3 & 48 & 22 \\
\hline Squinting & 10 & 53 & 10 \\
\hline Head Tilt & 23 & 50 & 0 \\
\hline Close to TV set & 59 & 13 & 1 \\
\hline Other eye disorders & 14 & 58 & 1 \\
\hline
\end{tabular}

Table 1

\begin{tabular}{|l|c|c|}
\hline \multicolumn{3}{|c|}{ Results of Ophthalmologic Screening } \\
\hline & Yes & No \\
\hline Head tilt & 4 & 69 \\
\hline Squinting & 3 & 70 \\
\hline Blepharitis & 14 & 59 \\
\hline Ptosis & 1 & 72 \\
\hline Scleral abnormalities & 2 & 71 \\
\hline Corneal abnormalities & 1 & 72 \\
\hline Iris defects & 2 & 71 \\
\hline Abnormal pupilary reaction to light & 0 & 73 \\
\hline $\begin{array}{l}\text { Cataract(s) or other lens } \\
\text { abnormalities }\end{array}$ & 2 & 71 \\
\hline Presence of esotropia & 27 & 46 \\
\hline Presence of exotropia & 9 & 64 \\
\hline Nystagmus & 13 & 60 \\
\hline
\end{tabular}

Table 2

disruptive behaviours. The remaining 61 patients had both near and distant visual acuity assessments. In most patients there was good correspondence (89\%) between near and distant visual acuity examinations. During the near vision screening patients often performed slightly better than during the distant vision screening. There were four patients in whom the near vision screening revealed markedly poorer vision than the distant vision screening and conversely in two patients the distant vision screening indicated significantly poorer results when compared with those of the near vision screening. 
For 58 individuals with Down syndrome, results of visual acuity examinations were available from both the private ophthalmologist and from screening done at the Child Development Center and there was good agreement in $91 \%$ between these two examinations. After reviewing the initial screening results and the records of previous eye examinations, it was decided that 18 children required more detailed ophthalmologic examinations.

Combining the results from screening as well as from previous and recent ophthalmologic evaluations, we obtained reliable and interpretable data of 68 individuals with Down syndrome: during the evaluation of visual acuity $71 \%$ of the children had either CSM or $20 / 50$ vision or better. These visual acuity results are based on vision with both eyes. Bilateral poor vision (20/50 or below) was found in 12 of 68 $(18 \%)$ patients, six of them had bilateral high refractive errors.

Of the 68 children with Down syndrome, 15 (22\%) were found to be amblyopic; five had associated strabismus, five had anisometropic amblyopia, and two had both strabismus and anisometropic amblyopia, and three patients did not have any associated findings.

\section{Discussion}

We engaged in this study using an unselected young population of individuals with Down syndrome enrolled in the Child Development Center at Rhode Island Hospital. The evaluation of the parents' questionnaire suggests that many parents are not too familiar with some of the ocular conditions in their children, although we used lay terms whenever possible and stayed away from medical jargon. Other parents may not have been told by their ophthalmologist details of their child's ocular problems. Only six of 73 of the children had not been seen by an ophthalmologist. Moreover, some of the conditions listed in the parents' questionnaire may have occurred in the past and were not present during the recent examination which explains the discrepancies between some of the results obtained from the questionnaire and the examinations. As noted in both Tables 1 and 2 , strabismus is more often observed in patients with Down syndrome than in the general population.

Most importantly, we found amblyopia to be present in $22 \%$ of children enrolled in this study. This prevalence figure is much higher than the $8.5 \%$ reported by Hiles, Hoyme and McFarlane (1974) and the 12.5\% observed by Jaeger (1980). Amblyopia in our study population was associated with strabismus in $33.3 \%$ and anisometropia in another $33.3 \%$, and $13.3 \%$ had both strabismus and anisometropia.

It is of note that 12 children in our study population were found to have bilaterally poor vision (20/50 or below). In nine of the 12 children bilateral high refractive errors were the cause of poor vision and in three of these children we were unable to determine a cause of their poor vision. When children with unilateral amblyopia were combined with children who have bilateral poor vision, a total of $40 \%$ of the children in our cohort had poor vision in one or both eyes. In addition, the prevalence of strabismus in our study was almost $50 \%$ which is higher than the frequency of strabismus of other reports which ranged from 23 to $44 \%$ (2-6). In the latter reports and as observed in our study, the majority of patients had esotropia.
The results of this study suggest that children with Down syndrome may be at greater risk for visual impairment than previously reported and that they may present with amblyopia either unilaterally or bilaterally. Therefore, it is important that these children be followed and treated appropriately to minimise that risk. Also, early treatment of strabismus and high refractive errors should reduce the level of amblyopia.

Because of the increased prevalence of amblyopia, frequently observed refractive errors, and other ovular disorders in youngsters with Down syndrome it is paramount that these children undergo ophthalmologic examination early in life and be followed and treated appropriately. Normal visual acuity is important for any child. However, if the child is mentally retarded, as most individuals with Down syndrome are, an additional handicap or sensory impairment may further limit the child's overall functioning and may prevent the child from participating in significant learning processes.

\section{Glossary}

Adnexa: The accessory structures of the eyeball.

Amblyopia: Diminished vision without structural abnormality of the visual pathway.

Anlsometropia:Marked inequality of refractive power of the two eyes.

Astigmatism: A condition in which the focussing process is asymmetrical.

Blepharitis: Inflammation of the eyelids.

Cataract:Opacity in the lens of the eye which may be partial or complete.

Canthl: The part which forms the angle at each end of the eyelids.

Esotropia:Squint, where the eyes point towards each other. Exotropia: Squint, where the eyes point away from each other.

Funduscopia: Examination of the fundus of the eye.

Fundus: The inner part of the back of the eye; it comprises the retina, optic disc and those parts of the choroid and sclera visible through the pupil.

Globe: The eyeball.

Hyperopia or Hypermetropia: Long sight. Difficulty in seeing an object near to the eyes.

Hyperpiasia: Any condition in which there is an increase in the number of cells in a part.

Keratoconus: Cone shaped cornea.

Myopia: Short sight. Difficulty in seeing an object at a distance.

Nystagmus:A condition in which the eyes are seen to move in a more or less rhythmical manner, from side to side, up and down or in a rotary manner.

Palpebra: Relating to the eyelids.

Ptosis: Drooping of the upper eyelid.

Sclera: The outer, opaque fibrous coat of the eyeball. It maintains the shape of the organ and provides attachment to the ocular muscles.

Snellen Charts: Chart used to test vision.

Strabismus: Squint.

\section{References}

Awaya,S. \& Miyake,S. (1988) Form vision deprivation amblyopia: further observations. Albrecht Von Graefes Archive der Klinischen Experimentellen Ophthalmologie, 225, 132136.

Brushfield,T. (1924) Mongolism. British Journal of Childhood Diseases, 21, 241-243. 
Caputo,A.R., Wagner,R.S. \& Reynolds,D.R. (1989) Down syndrome: Clinical review of ocular features. Clinical Pediatrics, 28, 355-358.

Catalano,R.A. (1992) Ophthalmologic concerns. In Pueschel,S.M. \& Pueschel,J.K. (Eds) Biomedical concerns in persons with Down syndrome. Paul H. Brookes Publishing Company.

Down,J.L. (1866) Observations on an ethnic classification of idiots. London Hospital Clinical Lectures and Reports, 3,259-262.

Gnad,H.D. \& Rett,A. (1979) Ocular signs in cases of Down's syndrome. Wiener Klinishe Wochenschrift, 91, 735-737. Hiles,D.A., Hoyme,S.H. \& McFarlane,F. (1974) Down's syndrome and strabismus. American Orthoptoptic Journal, 24, 63-68.

Jaeger,E.A. (1980) Ocular findings in Down's syndrome. Trans American Ophthalmological Society, 158, 808-845.

Lowe,R. (1949) Eyes in mongolism. British Journal of Ophthalmology, 33, 131-174.

Pueschel,S.M. (1981) Ophthalmological aspects in Down syndrome. Down's Syndrome: Papers and Abstracts for Professionals, 4, 4-7.

Rabinowicz,M. (1983) Amblyopia. In Harley,R.D. (Ed) Pediatric Ophthalmology Vol. I. W.B. Saunders Company, 293-342.
Rochels,R., Nover,A., \& Schmid,F. (1977) Ophthalmologic symptoms of Down's syndrome. Albrecht Von Graefes Archive der Klinischen Experimentellen Ophthalmologie, 205, 9-12.

Sequin,E. (1866) Idiocy and its treatment by the physiological method. William Wood and Co.

Shaw,D.E., Fiedler,A.R., \& Minshall,C. (1988). AmblyopiaFactors of influencing age of presentation. Lancet, 2, 207209.

Stager,D.R., Birch,E.E. \& Weakley,D.R. (1990) Amblyopia and the pediatrician. Pediatric Annals, 19, 301-305.

Tredgold,A.F. (1908) Mental deficiency (amentia). Bailliere, Tindall and Cox.

\title{
Advance Notice
}

\author{
A major International Conference on \\ Language and Cognitive Development in Down's Syndrome \\ will be held at \\ The University of Portsmouth, U.K. \\ 22nd - 25th September 1994
}

The keynote speakers are:

\begin{abstract}
Professor Jon Miller
University of Wisconsin, Madison, U.S.A.

Early language development $0-5$ years
\end{abstract}

Dr Anne Fowler

Bryn Mawr College \& Haskins Laboratories, New Haven

Connecticut, U.S.A.

Reading, phonemic awareness and phonological memory

\section{Dr Robin Chapman}

University of Wisconsin, Madison, U.S.A.

Language development in children and adolescents 5-20 years

\author{
Professor Jean Rondal \\ University of Liege, Belgium \\ Language development in adults with Down's \\ syndrome
}

Address for correspondence:

Child Development Centre,

Rhode Island Hospital,

Department of Pediatrics, 593 Eddy Street,

Providence, Rhode Island 02903

United States of America.
Dr Sue Gathercole

University of Lancaster, U.K.

The links between early language acquisition and cognition in infants with Down's syndrome

\section{Dr John Clibbens \\ University of Plymouth, U.K.}

The effects of teaching sign on the language development in children with Down's syndrome

\section{Dr David Messer}

University of Hertfordshire, U.K.

The links between early language acquisition and cognition in infants with Down's syndrome

\section{Sue Buckley}

University of Portsmouth, U.K.

The theoretical and practical significance of teaching literacy skills to children with Down's syndrome

The aim of the conference will be to bring together all the leading international researchers to discuss the current state of our knowledge and identify areas for future research. Poster presentations by participants will be welcomed. Deadline for submissions 31st March 1994. For further details, write to or fax the Sarah Duffen Centre: Belmont Street, Southsea, PO5 1NA, U.K. Fax no. 0705824265 